\title{
Identification of Genes Involved in Synaptogenesis Using a Fluorescent Active Zone Marker in Caenorhabditis elegans
}

\author{
Edward Yeh, ${ }^{1}$ Taizo Kawano, ${ }^{1}$ Robby M. Weimer, ${ }^{3}$ Jean-Louis Bessereau, ${ }^{3}$ and Mei Zhen ${ }^{1,2}$ \\ ${ }^{1}$ Samuel Lunenfeld Research Institute, Mount Sinai Hospital, Toronto, Ontario, M5G 1X5 Canada, ${ }^{2}$ Department of Microbiology and Medical Genetics, \\ University of Toronto, Toronto, Ontario, M5S 1A8 Canada, and ${ }^{3}$ Biologie Cellulaire de la Synapse, Ecole Normale Superieure, 75005 Paris, France
}

Active zones are presynaptic regions where synaptic vesicles fuse with plasma membrane to release neurotransmitters. Active zones are highly organized structurally and are functionally conserved among different species. Synapse defective-2 (SYD-2) family proteins regulate active zone morphology in Caenorhabditis elegans and Drosophila. Here, we demonstrate by immunoelectron microscopy that at C. elegans synapses, SYD-2 localizes strictly at active zones and can be used as an active zone marker when fused to green fluorescent protein (GFP). By driving expression of SYD-2::GFP fusion protein in GABAergic neurons, we are able to visualize discrete fluorescent puncta corresponding to active zones in living C. elegans. During development, the number of GABAergic synapses made by specific motoneurons increases only slightly from larvae to adult stages. In contrast, the number of SYD-2::GFP puncta doubles, suggesting that individual synapses accommodate the increasing size of their synaptic targets mainly by incorporating more active zone materials. Furthermore, we used this marker to perform a genetic screen to identify genes involved in the development of active zones. We recovered 16 mutants with altered SYD-2::GFP expression, including alleles of five genes that have been implicated previously in synapse formation or nervous-system development. Mapping of 11 additional mutants suggests that they may represent novel genes involved in active zone formation.

Key words: synapse; active zone; SYD-2; immunoelectron microscopy; GFP; genetic screen

\section{Introduction}

Synapse formation is a complex developmental process that is essential for the wiring of functional nervous systems. Although the morphology of chemical synapses varies among species, some fundamental structural features are common to all synapses (Garner et al., 2000; Dresbach et al., 2001; Zhen and Jin, 2004). One such feature is the active zone, a presynaptic region where synaptic vesicles fuse with the plasma membrane to release neurotransmitters. Active zones may vary in size and shape between synapses (Harlow et al., 2001; Phillips et al., 2001; Govind and Hirji, 2002; Gustafsson et al., 2002); however, they all contain electron-dense regions as sites of vesicle docking. Proper active zone formation is vital for synaptogenesis.

Received Dec. 7, 2004; revised Feb. 21, 2005; accepted March 1, 2005.

This work was supported by a research grant awarded to M.Z. from the Canadian Institutes of Health Research (CIHR). E.Y. was supported by a CIHR postdoctoral fellowship. R.M.W. was the recipient of an Institut National de la Santé et de la Recherche Médicale PosteVert fellowship. We thank J. Culotti and the members of the Zhen laboratory for comments on this manuscript and helpful discussions. We thank J. Dembowy and Y. Lui for assistance in generating some of the transgenic lines used in this study, S. Ng for mapping some of the identified mutants, Y. Wang for SYD-2 antibody generation, and T. Wu for help constructing the pJH191 plasmid. We thank M. Nonet (Washington University, St. Louis, MO) and J. Rand (Oklahoma Medical Research Foundation, University of Oklahoma, Oklahoma City, OK) for antibodies against UNC-10 and UNC-13. We thank B. Ackley and Y. Jin (University of California Santa Cruz, Santa $\mathrm{Cruz}, \mathrm{CA}$ ) for the pCZ529 plasmid. We are grateful for the schematic drawing of the GABAergic nervous system in Figure 6D, courtesy of E. Jorgensen (University of Utah, Salt Lake City, UT). Some strains for complementation tests were received from the Caenorhabditis Genetics Center.

Correspondence should be addressed to Dr. Mei Zhen, Samuel Lunenfeld Research Institute, Mount Sinai Hospital, 600 University Avenue, Toronto, Ontario, M5G 1X5 Canada. E-mail: zhen@mshri.on.ca.

R. M. Weimer's present address: Cold Spring Harbor Laboratory, 1 Bungtown Road, Cold Spring Harbor, NY 11724 DOI:10.1523/JNEUROSCI.4978-04.2005

Copyright $\odot 2005$ Society for Neuroscience $\quad$ 0270-6474/05/253833-09\$15.00/0
Through various approaches, some proteins that localize at or around active zone regions have been identified (Garner et al., 2000; Dresbach et al., 2001; Zhen and Jin, 2004). A technical difficulty in the biochemical purification of active zone materials is that components present in small quantities or with weak/ regulated association may escape detection. Forward genetic approaches have the potential to identify components that regulate active zone assembly and function. Recent genetic screens in Caenorhabditis elegans have expanded the list of genes that regulate synapse development (Schaefer and Nonet, 2001; Broadie and Richmond, 2002; Jin, 2002; Yamagata et al., 2003). Using a synaptobrevin (SNB)/SNB-1::GFP vesicle marker (Nonet, 1999), several novel genes were identified, including syd-2 (Zhen and Jin, 1999). Remarkably, mutations in some of these genes lead to little or mild alterations in behavior or pharmacological responses, suggesting that green fluorescent protein (GFP)-based screens are likely to be more sensitive to detect moderately abnormal synapses.

syd-2/liprin- $\alpha$ is required for regulating the size of active zones in C. elegans (Zhen and Jin, 1999). Drosophila Dliprin- $\alpha$ mutants also show defects in active zone specializations at glutamatergic neuromuscular junctions (Kaufmann et al., 2002). Several mammalian liprin- $\alpha$ proteins are expressed in the nervous system (Wyszynski et al., 2002). Thus, the role of syd-2/liprin- $\alpha$ in regulating active zone morphology is likely to be conserved. Synapse defective-2 (SYD-2)/liprin- $\alpha$ family proteins consist entirely of protein-interactive modules, and several candidate interactive proteins have been identified including active zone proteins 
CAST (CAZ-associated structurer protein) and RIM/UNC-10 (Rab3a-interacting molecule/uncoordinated-10), a kinesin motor KIF1A (Kinesin family member 1A), LAR (leukocyte antigenrelated)-type receptor tyrosine phosphatases, and a postsynaptic scaffolding protein, GRIP (glutamate receptor interacting protein) (Serra-Pages et al., 1998; Kaufmann et al., 2002; Schoch et al., 2002; Wyszynski et al., 2002; Kim et al., 2003; Ko et al., 2003; Shin et al., 2003).

We reasoned that a forward genetic screen using an active zone marker would preferentially uncover genes that regulate active zone assembly, and SYD-2 is an ideal candidate to generate such a marker. We first validate SYD-2 as an active zone marker and show that at $C$. elegans synapses, endogenous SYD-2 is strictly localized to presynaptic active zones. We describe a SYD-2::GFP marker that allows for direct visualization of active zones in living C. elegans. We identified 16 mutants that disrupt the normal expression of SYD-2::GFP. Two of these mutants may represent genes specifically involved in active zone formation. Our results suggest that the SYD-2::GFP marker may be a powerful tool to study active zone formation and regulation.

\section{Materials and Methods}

Genetic methods and alleles. Standard methods were used for culturing and handling animals (Brenner, 1974). juIs1 (Punc-25-SNB-1::GFP) has been described previously (Zhen and Jin, 1999). pJH23 (Punc-25-SYD-2::GFP) was coinjected with lin-15 marker into lin15(n765), integrated into the C. elegans genome, and out-crossed five times against wild-type $\mathrm{N} 2$ strain to generate $h p I s 3$. pCZ529 (Punc-25-SNB-1::CFP; kindly provided by B. Ackley and Y. Jin, University of California Santa Cruz, Santa Cruz, CA) was coinjected with lin-15 marker into lin-15(n765) animals to generate hpEx20. pJL37 (Punc-47-SNB-1::CFP) or pJH191 (Punc-25-SNB-1::DsRed) were coinjected with RF4 marker into wild-type animals to generate transgenic lines $h p E x 85$ and $h p E x 86$. pJH191, pJL37, and pCZ529 give similar expression patterns, except that the signal from pJH191 is much weaker than pJL37 and pCZ529. pJH355 (Punc-25-DsRed) was coinjected with Pttx-3-GFP into $h p I s 3$ to generate $h p I s 3 ; h p E x 567$.

Constructs. pJH23 (Punc-25-SYD-2::GFP) was generated by inserting a $1 \mathrm{~kb}$ GFP fragment into the BamHI site of the pJH52 (Punc-25-SYD-2) clone. pJH355 was generated as follows. PCR primers (OZM342 and OZM343) were used to amplify the coding sequence for DsRed from DsRed2C1 (Clontech, Palo Alto, CA). These primers introduce a 5' BamHI site, a 3' BglII site, and a stop codon immediately after the last coding amino acid. The resulting PCR product was inserted into the BamHI site of a plasmid (pJH21) that puts it behind the Punc-25 promoter plus the first eight amino acids of UNC-25.

Whole-mount antibody staining. Animals were fixed in $2 \%$ paraformaldehyde for $4 \mathrm{~h}$ and stained as described previously (Zhen and Jin, 1999). GFP puncta in juIs 1 and $h p I s 3$ animals were preserved by the fixation procedure and thus can be visualized without antibody staining. Polyclonal antibodies against SYD-2 protein were generated by injecting 6 XHis SYD-2 fusion protein into rats and rabbits (Covance, Berkeley, CA) and used at 1:10 and 1:40 dilutions, respectively. Rabbit anti-UNC-10 (M. Nonet, University of Washington, St. Louis, MO) and anti-UNC-13 (J. Rand, University of Oklahoma, Oklahoma City, OK) antibodies were used at 1:12,000 and 1:50 dilutions, respectively. Cyanine5 (Cy5)-conjugated donkey anti-rat, Cy3conjugated donkey anti-rabbit, and FITC-conjugated donkey anti-chicken secondary antibodies (Jackson ImmunoResearch, West Grove, PA) were used at 1:400 dilution.

Immunoelectron microscopy. Wild-type $\mathrm{N} 2$ animals were immobilized by high-pressure freezing, dehydrated by freeze substitution, and embedded as described previously (Rostaing et al., 2004), with the following exceptions. After high-pressure freezing, physically immobilized animals were dehydrated at $-90^{\circ} \mathrm{C}$ in $0.1 \% \mathrm{KMnO}_{4}$ in acetone for $3 \mathrm{~d}$ before embedding in Lowicryl HM20. Thin $(50 \mathrm{~nm})$ sections were immobilized on formvar-coated slot grids, stained with rabbit anti-SYD-2 (1:50 dilu- tion), revealed with $10 \mathrm{~nm}$ gold-conjugated goat anti-rabbit antibodies, and imaged via a Phillips electron microscope equipped with a Gatan DV 300W digital camera.

Quantifying puncta. Animals to be quantified were reared at $22^{\circ} \mathrm{C}$. Live animals were immobilized by placing large numbers of animals $(>25)$ onto dried 2\% agarose pads with a drop of M9. Using these conditions, some worms can withstand the pressure of the coverslip while being immobilized sufficiently for imaging. Imaging was done using an Axioplan 2 microscope (Zeiss, Thornwood, NY) equipped with an ORCA-ER digital camera (Hamamatsu, Hamamatsu City, Japan) operated by Openlab software (Improvision, Lexington, MA). Z-plane scans were taken of the dorsal cord regions of animals. Puncta were counted from the Z-plane images. $p$ values were calculated using the Mann-Whitney $U$ test (Instat 3; Graph Pad, San Diego, CA).

To determine the degree of colocalization between SYD-2 and UNC10/UNC-13 immunostaining, Z-plane scans were taken of dorsal cord regions alternating between the appropriate emission channels. Scanning through the Z-plane images in both separate channels, SYD-2 puncta were counted and scored as colocalizing with UNC-10/UNC-13 if any overlap in immunostaining was detected within the same Z-plane. A similar approach was used to determine SYD-2::GFP colocalization with UNC-10.

Ethylmethane sulfonate mutagenesis screen. sem-4; hpIs3 worms were mutagenized with ethylmethane sulfonate (EMS) as described previously (Brenner, 1974). Young larval (L2 and L3 stages) animals were placed on $2 \%$ agarose pads on microscope slides under a coverslip and screened on a Zeiss Axioplan 2 fluorescence microscope equipped with a $63 \times$ objective. Candidate mutants were recovered from the microscope slide and allowed to pass through several generations before rescreening. $\mathrm{Mu}-$ tants were kept if the mutant phenotype could be detected after outcrossing to N2.

\section{Results}

\section{SYD-2 localizes to presynaptic active zones in C. elegans}

Previous studies have implicated SYD-2 as an active zone component that is required for the morphology and function of active zones in C. elegans (Zhen and Jin, 1999). However, studies in mammalian cultured neurons suggest that vertebrate liprin- $\alpha$ protein localization may not be restricted to presynaptic compartments (Wyszynski et al., 2002). To determine whether SYD-2 would be a suitable candidate as an active zone marker, we regenerated specific polyclonal antibodies against SYD-2 (data not shown). We then performed double-antibody staining against endogenous SYD-2 and two known active zone components, UNC-10/RIM and UNC-13/Munc-13, that are required for the fusion of synaptic vesicles in C. elegans (Richmond et al., 1999; Koushika et al., 2001). Double-antibody staining against SYD-2 and UNC-10 revealed that SYD-2 colocalizes with UNC-10 as discrete puncta of $\sim 200-400 \mathrm{~nm}$ in diameter along the dorsal, ventral, and lateral nerve cords (Fig. $1 A-C$ ). Double-antibody staining against SYD-2 and UNC-13 revealed that SYD-2 also colocalizes with UNC-13 (Fig. $1 D-F$ ). In both cases, the degree of colocalization was very high but not complete $[92.9 \pm 2.3 \%$ for UNC-10 $(n=5)$ and $83.1 \pm 6.2 \%$ for UNC-13 $(n=6)]$. Some SYD-2 puncta appear to stain with varying amounts of UNC-10 or UNC-13 and vice versa, and in some cases, SYD-2 puncta do not costain at all with UNC-10 or UNC-13.

To determine the ultrastructural localization of SYD-2 within the synapse, we performed immunoelectron microscopy with rabbit antibodies against SYD-2. Electron micrographs reveal that SYD-2 positive gold particles are closely associated with the electron-dense specializations within active zone regions at both neuromuscular junctions $(n=9)$ (Fig. $2 A, B)$ and neuron-neuron synapses ( $n=7$; data not shown). Specifically, measurements within the presynaptic and postsynaptic axes (Fig. 2C) demonstrate that the majority of gold particles accumulate within the 


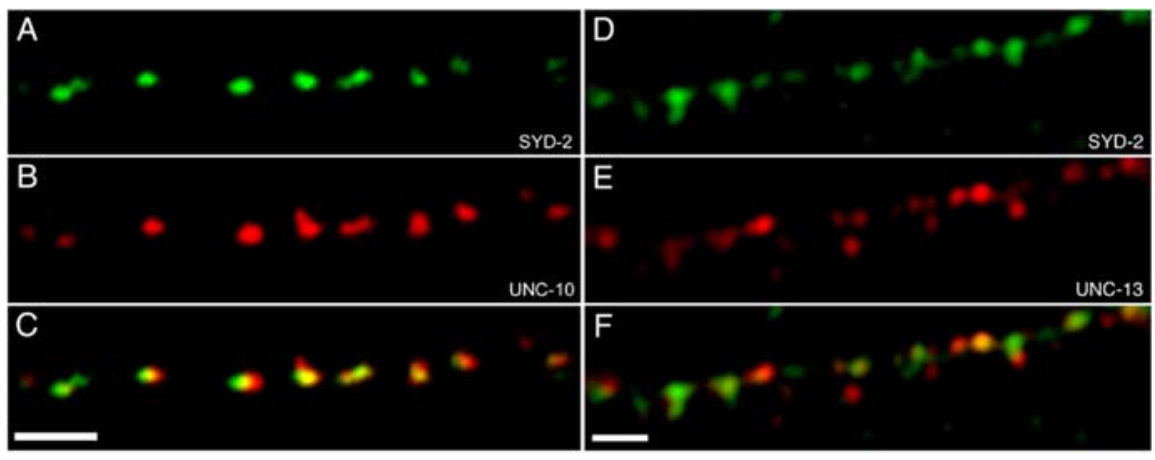

Figure 1. SYD-2 localizes to presynaptic active zones. A-C, A comparison of SYD-2 immunofluorescence (green) and UNC-10 immunofluorescence (red) suggests that the two proteins colocalize to discrete puncta in the dorsal nerve cord. $\boldsymbol{D}-\boldsymbol{F}$, Similarly, a comparison of SYD-2 immunofluorescence (green) and UNC-13 immunofluorescence (red) suggests that SYD- 2 and UNC-13 colocalize. The overlay of the two channels reveals some partial colocalization and possible lack of equal stoichiometry. Scale bars, $1 \mu \mathrm{m}$.

presynaptic terminal, on average $12 \mathrm{~nm}$ from the presynaptic plasma membrane. Laterally, gold particles accumulate around the electron-dense presynaptic specializations (Fig. 2C). The average distance of a gold particle to the center of the nearest presynaptic specialization was $17 \mathrm{~nm}$ (Fig. 2 D, E; supplemental Fig. 1 , available at www.jneurosci.org as supplemental material). This confirms that at $C$. elegans synaptic junctions, SYD-2 is strictly localized at presynaptic termini, tightly associated with the electron-dense specializations in active zones.

\section{SYD-2::GFP expression in GABAergic motoneurons reveals presynaptic active zones}

A DNA construct that expresses a SYD-2::GFP fusion protein was generated by an in-frame insertion of GFP at the very $\mathrm{N}$ terminus of the SYD-2 open reading frame. This fusion protein is functional because its expression by a pan-neural promoter fully rescues the sluggish movement of syd-2 mutant animals. To visualize active zones in single synapses, we limited the expression of the SYD-2::GFP fusion protein to a subset of motoneurons by using the $u n c-25$ promoter to drive expression in the GABAergic VD and DD motoneurons (Jin et al., 1999). Visualization of a transgenic strain carrying the Punc-25-SYD-2::GFP construct $h p I s 3$ revealed that the SYD-2::GFP fusion protein is localized to discrete puncta of 200-400 $\mathrm{nm}$ in diameter along the dorsal and ventral nerve cord (see Fig. $5 A, B$ ). In addition, strong GFP fluorescence is seen in VD and DD cell bodies (data not shown). The cell body localization is most likely caused by overexpressed SYD-2::GFP fusion proteins that fail to be transported, because endogenous SYD-2 is never detected in motoneuron cell bodies (Zhen and Jin, 1999).

Immunofluorescent staining with antibodies against the active zone protein RIM/UNC-10 in hpIs3 animals shows that SYD-2::GFP puncta localize to active zones. A total of $79.8 \pm$ $5.3 \%(n=9)$ of SYD-2::GFP puncta in the dorsal cord colocalize with UNC-10 (Fig. 3A-C). As with endogenous SYD-2/UNC-10 colocalization, not all SYD-2::GFP are stained with UNC-10. In addition, we frequently observed that very bright SYD-2::GFP puncta had little or no UNC-10 staining (Fig. 3C). Thus, the decrease in colocalization of SYD-2::GFP may be attributable to the exclusion of UNC-10 antibody to the active zone by GFP. To further confirm that SYD-2::GFP puncta localize to active zones, a Punc-25-SNB-1::CFP, Punc-25-SNB-1::DsRed, or Punc-47-SNB-1::CFP construct was introduced into $h p I s 3$ animals. Synaptobrevin/SNB-1 is a synaptic vesicle protein, and SNB-1::GFP protein allows the direct visualization of vesicle pools accumulated at presynaptic termini (Nonet, 1999). When introduced into wild-type animals, cyan fluorescent protein (CFP) or DsRed-tagged SNB-1 shows the same protein expression and localization as GFP-tagged SNB-1 when expressed under the $u n c-25$ or $u n c-47$ promoter, which also drives expression specifically in VD and DD motoneurons (McIntire et al., 1997) (compared with juIs1; data not shown). Although GFP and DsRed/CFP show some leakage into spectrum of each other, when introduced into hpIs3 animals, SNB-1::CFP/DsRed puncta are distinctively larger than SYD-2::GFP puncta, and with the intensity difference, we can unambiguously distinguish between the two puncta by simultaneous live imaging in both channels. It is clear that all visible SYD-2::GFP puncta are associated with SNB-1::CFP puncta (Fig. 3D).

\section{Expression and localization of SYD-2::GFP in known synapse-defective mutants}

To test whether the SYD-2::GFP marker can reflect changes in active zone morphology and distribution, we examined the expression pattern of hpIs 3 in a few C. elegans mutants with known active zone distribution defects. Two of these mutants, $r p m-1$ and $f_{s n-1}$, function in a ubiquitination pathway to regulate synapse formation (Schaefer et al., 2000; Zhen et al., 2000; Liao et al., 2004). In either mutant, some GABAergic synapses aggregate with clustered active zone specializations in some regions, and there are fewer presynaptic specializations in other axonal regions. We observed that the expression and localization of the SYD-2::GFP marker indeed was altered in these mutants and reflected their previously characterized defects. SYD-2::GFP puncta cluster in some axonal regions and show gaps in other regions where no obvious puncta can be detected (Fig. $4 B, f_{s n}-1$ ) (rpm-1 data not shown). In addition, an allele of $r p m-1$ was recovered from our mutagenesis screen (see below). unc-104 mutants are defective in kinesin function, and thus synaptic vesicles fail to be transported to presynaptic termini. Ultrastructural analysis of unc-104 mutants shows an $80 \%$ reduction in synapse numbers (Hall and Hedgecock, 1991; Otsuka et al., 1991). Consistently, there were far fewer SYD-2::GFP puncta in unc-104 mutants when compared with wild type (Fig. 4C). Similarly, we see defects in synapse formation in unc-116 mutants, which are also defective in kinesin function (Fig. 4D). syd-1 mutants also display defects in synapse formation as observed by defective SYD-2::GFP localization resulting in puncta clustering and gaps (Fig. $4 E$ ). The syd-1 gene is required for the polarity of DD and VD motoneurons (Hallam et al., 2002). Loss of syd-1 function leads to an ectopic innervation of dorsal body wall muscles by VD neurons, in addition to the normal innervation by DD neurons. We also isolated an allele of syd-1 from our mutagenesis screen (see below).

The number of SYD-2::GFP puncta per presynaptic terminal increases during $C$. elegans development

We examined the expression pattern of the SYD-2::GFP active zone marker ( $h p I s 3)$ during development and compared it with that of GABAergic synapses visualized by the vesicle marker 

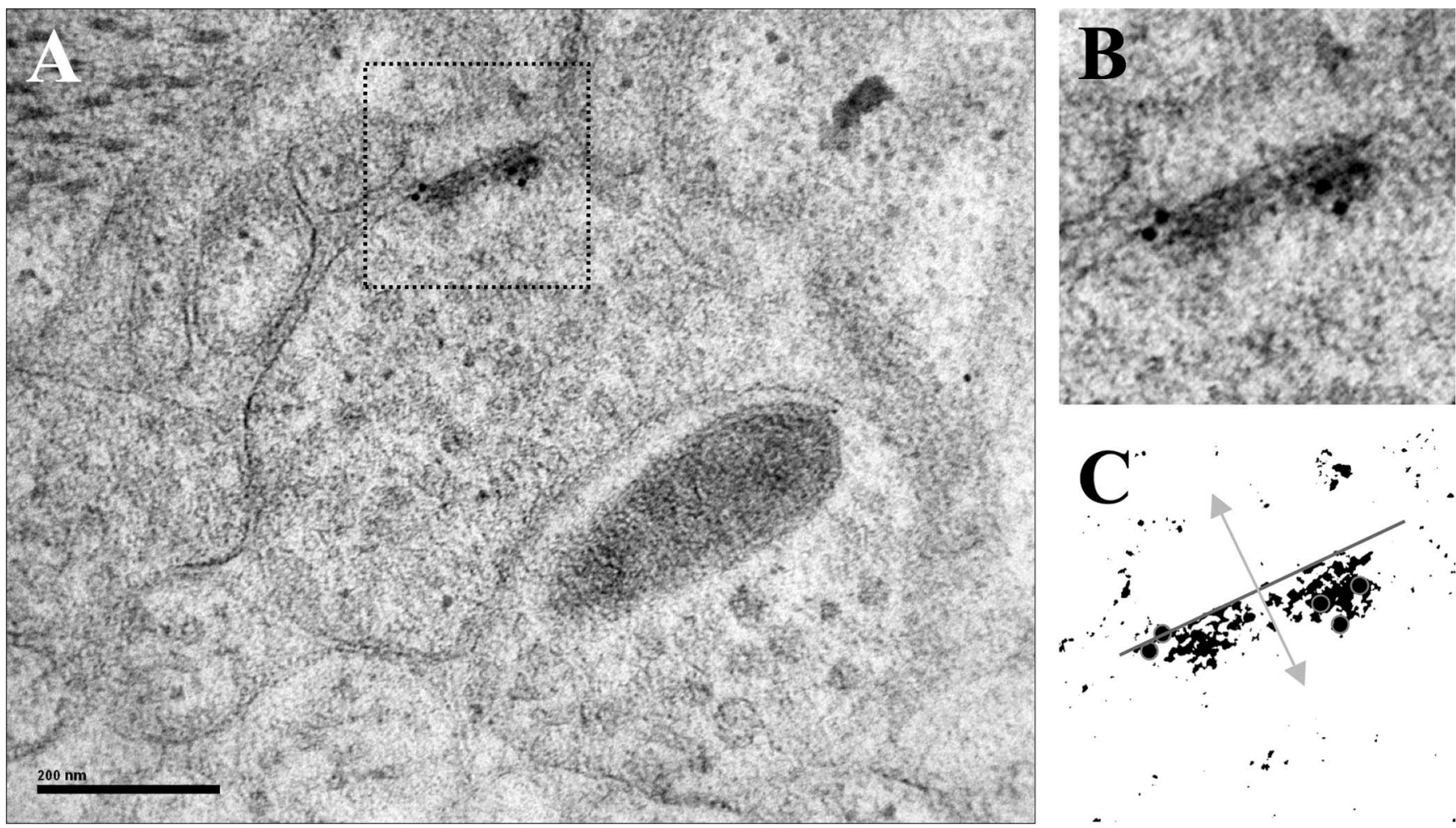

D

pre-post axis distribution

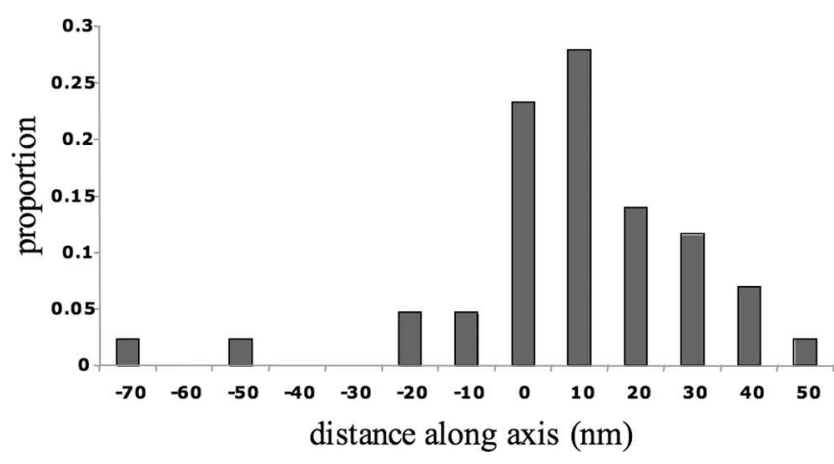

Figure 2. SYD-2 immunoelectron staining at chemical synapses. $A$, An electron micrograph shows SYD-2 immunolabeling of electron-dense active zones. A higher magnification of the outlined region is shown in $\boldsymbol{B}$, whereas the same image is shown schematized in $\boldsymbol{C}$. The axis in which distances of gold particles were measured (arrow) from the electron-dense region of the active zone (line) is shown, as well as active zone-associated gold particles (circles). $\boldsymbol{D}, \boldsymbol{E}$, Analyses of the distances of gold particles along the presynaptic (pre) and postsynaptic (post) axes show that the majority of SYD-2 gold particles are within $12 \mathrm{~nm}$ of the presynaptic active zone.

SNB-1::GFP ( juIs1). A comparison of the distribution of $h p I s 3$ and juIs1 suggests that the ratio between the number of SYD-2::GFP fluorescent puncta versus synaptic vesicle puncta changes when animals grow (Fig. 5). To gain insight into how synapse formation proceeds in C. elegans, we quantified the expression of $h p I s 3$ and juIs 1 markers at different developmental stages by two different methods.

In the first method, the total number of hpIs3 puncta was counted in a defined dorsal cord region where synapses are made mainly by the DD5 motoneuron. This region is unambiguously identified between the commissures of two neurons, VD10 and VD12, which are labeled by the axon marker Punc-25-DsRed (Fig. $6 B, D$ ). The number of $h p I s 3$ puncta increases by approximately twofold from the second larval (L2) to adult stage within this region (L2 and adult; $p<0.0001$ ), whereas the number of
juIs1 puncta increases by $\sim 29 \%$ (L2 and adult; $p<0.0001$ ) (Fig. $6 E$, dark filled bars). Because the physical length of this region increases while the animals grow during development (in all samples quantified, this distance increases by $\sim 3.2$-fold from L2 to adult; $p<0.0001$ ), this result suggests that the motoneuron responsible for innervating this area copes with the increase in synaptic area mainly by increasing the amount of active zone components and/or strength in existing synapses.

Quantification was also performed by counting the total number of hpIs3 and juIs1 puncta within a fixed distance $(50 \mu \mathrm{m})$ in similar areas of the dorsal cord at various developmental stages. Unlike the first method, the fixed distance reflects a continuously decreasing fraction of the innervated region by specific motoneurons while animals grow. We found that within a fixed distance, the total number of juIs1 puncta decreases by $\sim 44 \%$ as animals 

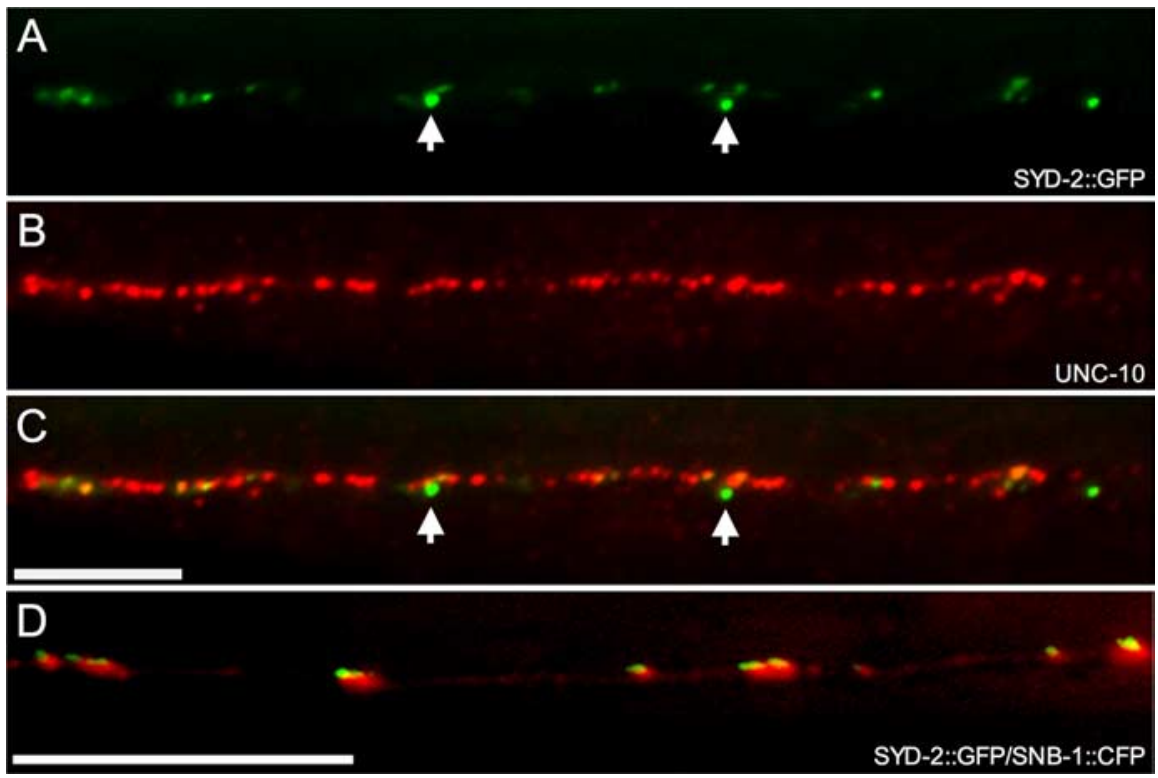

Figure 3. SYD-2::GFP localizes to active zones in vivo. $A-C$, Antibody staining against UNC-10 (red) shows that many SYD-2::GFP puncta (green) colocalize with UNC-10. Many UNC-10 puncta do not have a corresponding SYD-2::GFP puncta because endogenous UNC-10 labels all active zones in the dorsal cord, whereas SYD-2::GFP expression is limited to GABAergic motoneurons. However, some very bright SYD-2::GFP have little or no UNC-10 staining (arrows). D, Live SYD-2::GFP puncta (green) associate with SNB-1::CFP puncta (red), indicating that SYD-2::GFP puncta represent active zones in vivo. Scale bars, $5 \mu \mathrm{m}$.
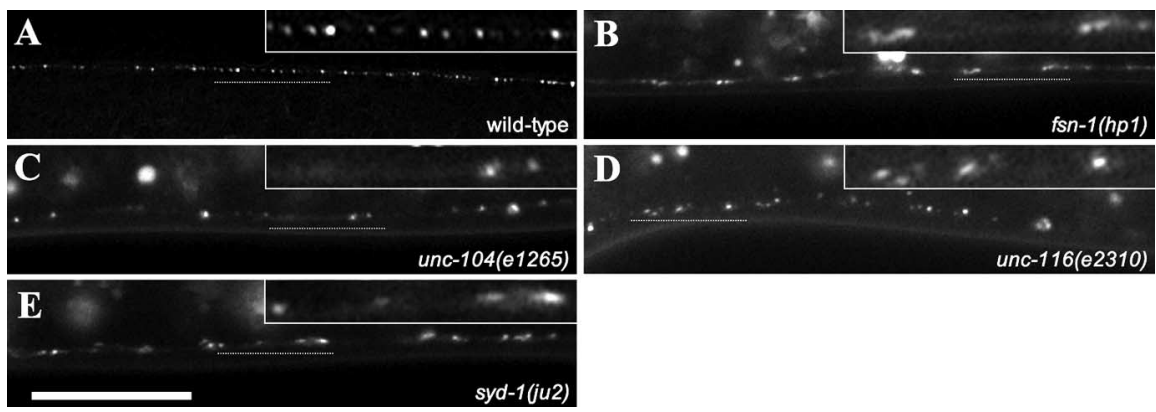

Figure 4. SYD-2::GFP expression and localization in known synapse-defective mutants. All images are of dorsal cords from L2 stage animals (insets show a higher magnification of a region of the dorsal cord marked by the dotted line). $A$, Wild-type $h p / s 3$ SYD-2::GFP expression and localization. $\boldsymbol{B}, \boldsymbol{E}$, fsn-1 (hp 1) and syd-1 ( ju2) show clustered puncta with gaps. C, unc-104 (e1265) mutants display less active zone puncta. $\boldsymbol{D}$, unc-116 (e2310) mutants have clustered puncta and overall disorganized puncta distribution. Scale bar, $10 \mu \mathrm{m}$.
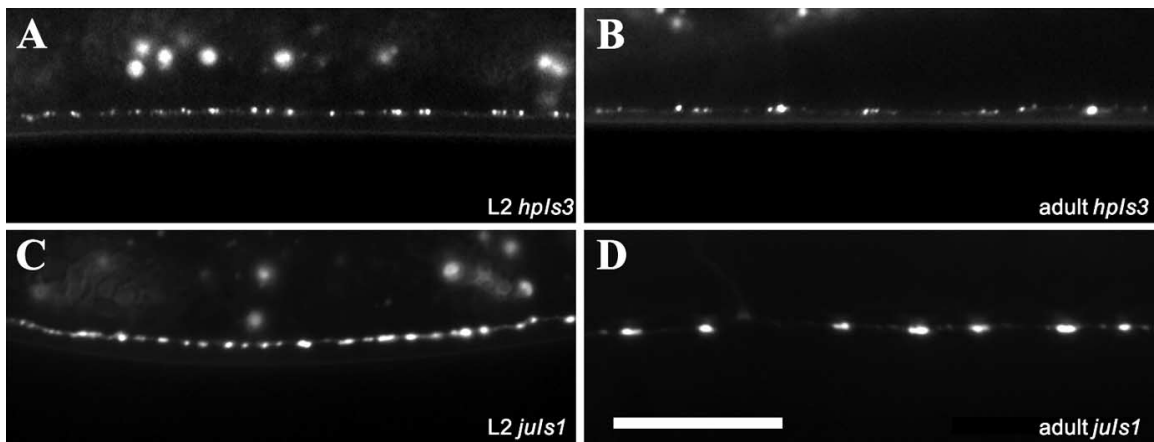

Figure 5. A comparison of SNB-1::GFP and SYD-2::GFP expression and localization during development. $A, B, h p / s 3$ puncta at L2 (second larval stage) and adult. At later developmental stages, hpls3 puncta increase in number per synaptic termini. A comparison of juls 1 puncta at $L 2$ and adult stages $(\boldsymbol{C}, \boldsymbol{D})$ shows that the spacing between synapses increases as animals grow, resulting in a decrease in synapse density along the dorsal cord. The size of juls 1 puncta is also slightly increased. Scale bar, $10 \mu \mathrm{m}$. proceed in development (L2 and adult; $p<0.0001)$. This suggests that during development, the spacing of synapses increases and the density of synapses decreases (Fig. $6 E$, shaded bars). In contrast, the total number of $h p I s 3$ puncta decreases by only $18.8 \%$ (L2 and adult; $p=0.0001$ ), suggesting that active zone density does not decrease in accordance with decreasing synapse density during development. Additional hpIs3 puncta have to be added during development to maintain the active zone density within the fixed distance.

To determine whether the relatively even density of SYD-2::GFP observed during development was an artifact of exogenous SYD-2 expression, we quantified the number of endogenous SYD-2 and UNC-10 puncta in larval and adult animals using immunofluorescent staining (supplemental Fig. 2, available at www.jneurosci.org as supplemental material). The distribution of UNC-10 and SYD-2 is fairly even along the dorsal nerve cord throughout development. The number of endogenous SYD-2 puncta observed per $50 \mu \mathrm{m}$ is $91.4 \pm 5.6$ in L2 $(n=10)$ and $88.5 \pm 7.6$ in adults $(n=10)$; the number of endogenous UNC-10 puncta observed per $50 \mu \mathrm{m}$ is $87.1 \pm 8.7$ in L2 $(n=7)$ and $85.4 \pm 11.3$ puncta in adults $(n=6)$. This suggests that the density of endogenous SYD-2 and UNC-10 does not change significantly between L2 and adult (SYD-2, $p=0.3840$; UNC-10, $p=0.7750)$. Although most synapses along the dorsal cord are a combination of GABAergic and cholinergic neuromuscular junctions, this is consistent with our previous observation that the density of active zone components represented by the hpIs3 marker does not change significantly during development. Therefore, both quantification methods suggest that, at least in GABAergic motoneurons, synaptic growth during development is mainly accomplished by increasing the amount of active zone material within a relatively fixed number of synapses, which predicts an increase in the release capacity with growth/development.

\section{A genetic screen to identify genes}

involved in synaptogenesis

Because the SYD-2::GFP marker can be used to visualize and analyze the development of active zones in vivo, we performed an EMS mutagenesis screen with $h p I s 3$ to identify genes that alter the morphology or development of active zones when mutated. We performed a clonal recessive screen of $3500 \mathrm{mu}$ tagenized haploid genomes. Sixteen mutants were isolated as candidates for 

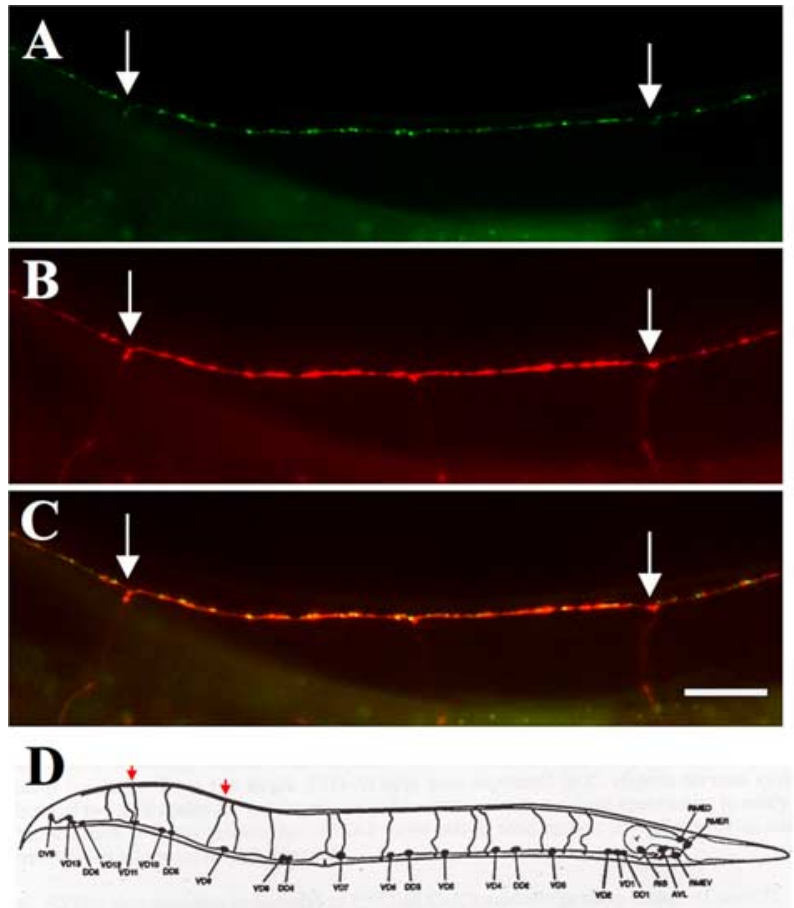

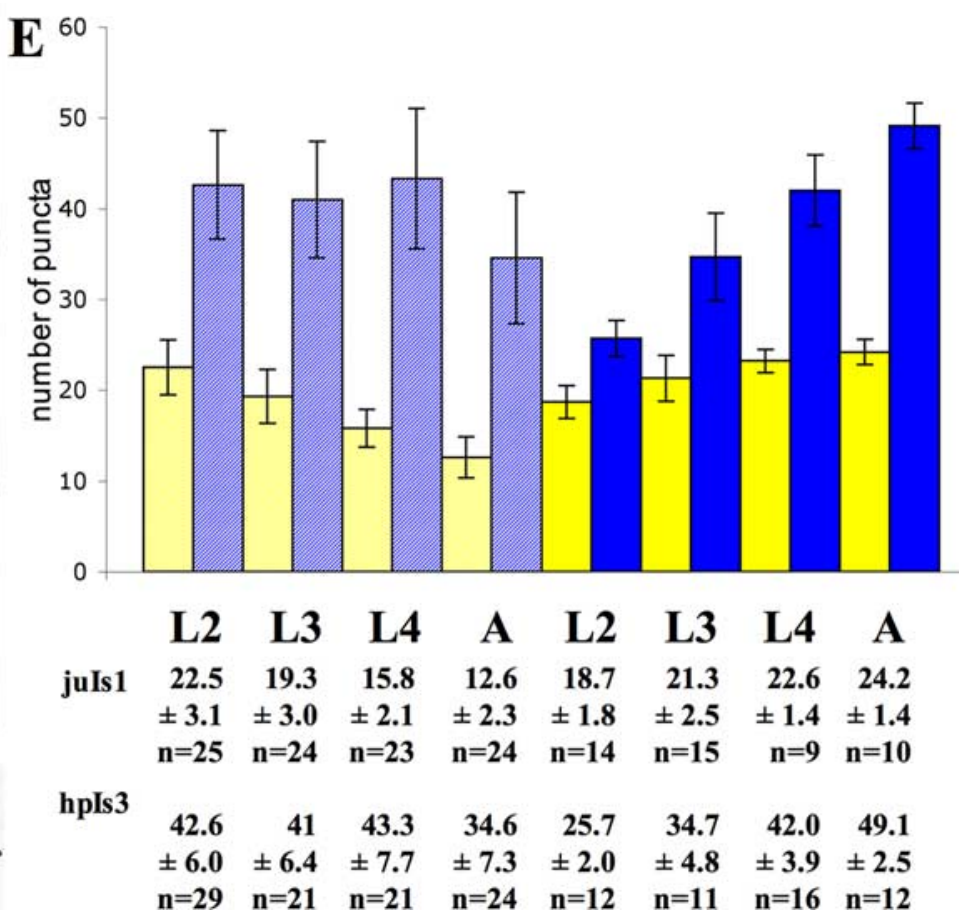

Figure 6. Quantification of SNB-1::GFP and SYD-2::GFP puncta during development. Using Punc-25-DsRed to label GABAergic neurons, SYD-2::GFP ( $h$ pls3) puncta were counted in the dorsal cord region between the commissures of motoneurons VD10 and VD12. $A-C$, Examples of DsRed-labeled commissures meeting the dorsal cord (arrows). Scale bar, $10 \mu m$. D, Schematic representation of this region (courtesy of E. Jorgensen). $\boldsymbol{E}$, The results of SNB-1::GFP ( juls 1) and SYD-2::GFP (hpls3) puncta quantification within this region (as shown in $\boldsymbol{A}-\boldsymbol{D}$ ) are illustrated by dark filled bars. Puncta were also quantified by counting the number of puncta within a $50 \mu \mathrm{m}$ distance at different stages during C. elegans development. These results are shown as shaded bars. Numbers for juls 1 puncta are shown in yellow, and numbers for $h p / s 3$ puncta are shown in blue.

Table 1. Mutants recovered from a screen for synapse-defective mutants

\begin{tabular}{|c|c|c|c|c|}
\hline Allele & SYD-2::GFP phenotype & Visible phenotype & SNB-1::GFP phenotype & Gene \\
\hline \multicolumn{5}{|l|}{ I } \\
\hline hp96 & Diffuse & Wild type & ND & ND \\
\hline hp151, hp155 & Highly diffuse, big puncta & Hyperactive & ND & goa-1 \\
\hline \multicolumn{5}{|c|}{ 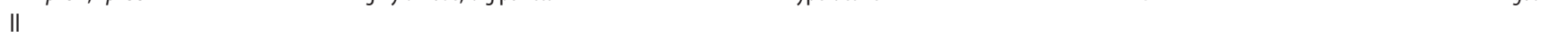 } \\
\hline$h p 45$ & Diffuse, clustering, gaps & Backward Unc & Yes & syd-1 \\
\hline hp104 & Diffuse, big GFP haze & Mild Unc & ND & ND \\
\hline \multicolumn{5}{|c|}{ 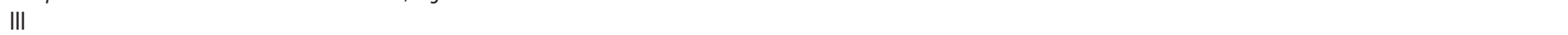 } \\
\hline hp34 & Diffuse axon, gaps & Wild type & ND & ND \\
\hline \multicolumn{5}{|c|}{ 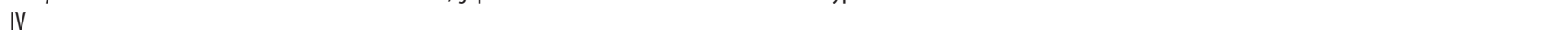 } \\
\hline hp102 & Diffuse puncta, gaps & Loopy Unc & Yes & ND \\
\hline hp149 & № GFP expression & Unc & ND & unc- 30 \\
\hline \multicolumn{5}{|l|}{ V } \\
\hline hp129 & Gaps & Wild type & No & ND \\
\hline hp97 & Diffuse axon, clustering, gaps & Slightly Dpy & Yes & rpm-1 \\
\hline \multicolumn{5}{|c|}{ (5) } \\
\hline hp77 & Gaps & Wild type & ND & ND \\
\hline hp30 & Diffuse axon, some gaps & Wild type & ND & ND \\
\hline hp121 & Less puncta & Kinker Unc & No & ND \\
\hline hp150, hp153 & Diffuse, clustering, gaps & Unc & ND & unc- -3 \\
\hline hp157 & Highly diffuse puncta & Float on buffer & ND & ND \\
\hline
\end{tabular}

Mutants are listed by chromosome. Unc, Uncoordinated locomotion; Dpy, dumpy; ND, not determined.

genes involved in active zone formation or other aspects of synapse development (Table 1).

The phenotypes of the mutants recovered from this screen can be described generally using different categories. One phenotypic category is a reduction in the number of $h p I s 3$ puncta and/or the presence of large regions along axons devoid of puncta (referred to as "gaps" in Table 1 and in Figs. 1-7). Mutants hp150, hp129, hp102, and $h p 121$ display this phenotype (Fig. $7 E-H$ ). Another phenotypic category is the diffuse localization of the SYD-2::GFP marker within the presynaptic terminal and/or along the axon. Several mutants display different degrees of severity of diffusion (Table 1). Among them, $h p 151$ and $h p 155$ show significant increase of SYD-2::GFP puncta size and intensity at the presynaptic terminal (Fig. $7 D$ ), whereas $h p 45$ and $h p 97$ show varying degrees of diffuse SYD-2::GFP localization along the axon and within the synaptic terminal (Fig. $7 B, C)$. A third phenotypic category is the clustering of $h p I s 3$ puncta, such as in $h p 102$ (Fig. 7G), which also leads to the diffuse appearance of SYD-2::GFP within presynaptic termini. Many mutants display a 

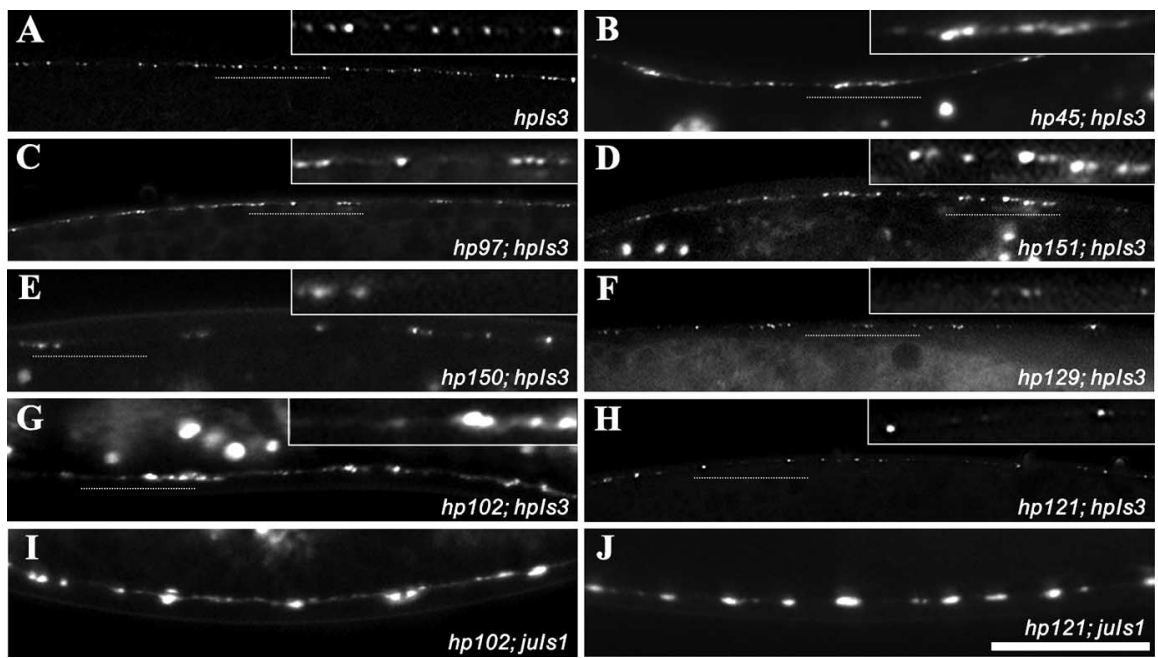

Figure 7. Phenotypes of mutants recovered from SYD-2::GFP screens. $\boldsymbol{A}-\boldsymbol{H}$, Images are of dorsal cords from $\mathrm{L} 2$ stage animals (insets show a higher magnification of a region of the dorsal cord marked by the dotted line). $\boldsymbol{A}$, Wild-type hpls3 SYD-2::GFP expression and localization. $h p 45, h p 97$, and $h p 151$ show varying degrees of diffuse localization around presynaptic termini and axons ( $\boldsymbol{B}-\boldsymbol{D}$, respectively). $h p 150, h p 129$, hp 102, and $h p 121$ show less puncta and gaps in the dorsal cord where there are no SYD-2::GFP puncta ( $\boldsymbol{E}-\boldsymbol{H}$, respectively). In addition, some mutants show combinations of these phenotypes such as $h p 45$, $h p 150$, hp129, and hp 102.I, J, SNB-1::GFP ( juls1) expression and localization in the dorsal cord of adults appears to be disrupted in $h p 102$ but fairly normal in $h p 121$ (compare with Fig. 5D). All panels are at the same magnification. Scale bar, $10 \mu \mathrm{m}$.

combination of all of these phenotypes. For example, $h p 129, h p 102$, $h p 97$, and $h p 45$ display fewer puncta and varying degrees of clustering and diffuse SYD-2::GFP localization at different regions along the dorsal cord.

We have examined SNB-1::GFP expression and localization in $h p 45, h p 97, h p 102, h p 121$, and $h p 129$ mutants. $h p 45, h p 97$, and hp102 display defects in SNB-1::GFP localization that correlate with the SYD-2::GFP phenotype, such as diffusion and clustering (Table 1; Fig. 7I, hp102). However, $h p 121$ and $h p 129$ have fairly normal SNB-1::GFP distribution (Fig. 7J, hp121), suggesting that these genes may specifically affect the composition, morphology, or structure of active zones.

Of the mutants recovered from our screen, five are allelic to known genetic loci. $h p 97$ animals show gaps and clustering of active zone puncta and are also slightly shorter in body length. hp97 is allelic to $r p m-1(j u 44)$. This is further confirmed as SNB-1::GFP expression and localization in $h p 97$ revealed synaptic defects similar to those described previously for $r p m-1$. Another mutant, $h p 45$, displays clustered active zone puncta and is allelic to syd-1(ju2). Ectopic clustering of active zone puncta along the dorsal cord is consistent with the phenotype described for syd-1 mutants. $h p 149$, which lacks SYD-2::GFP expression in the VD and DD motoneurons (data not shown), is allelic to unc30. unc-30 encodes a transcription factor required for transcription of GABAergic-specific genes, including unc-25 (McIntire et al., 1993; Jin et al., 1994; Eastman et al., 1999). Both $h p 151$ and $h p 155$ are allelic to goa-1, the Go $\alpha$ subunit that was reported previously to decrease the abundance of UNC-13 protein at release sites in C. elegans (Nurrish et al., 1999). The increased size of SYD-2::GFP puncta in goa-1 mutants is consistent with the report and further suggests that Go $\alpha-1$ may negatively control the size of active zones. $h p 150$ and $h p 153$ are two alleles of $u n c-3$, which encodes a novel transcription factor required for nervous system development and axon guidance (Prasad et al., 1998). In unc-3 mutants, many active zone puncta are clustered and SYD-2::GFP is diffusely localized along the axon. The SYD-2::GFP defects in unc-3 mutants are unlikely to be solely secondary consequences of axonal abnormalities. Although axon guidance defects are present in low penetrance in $h p 150$ and $h p 153$, severe SYD-2::GFP defects were observed in regions where axons were present and appeared normal (Fig. 7E). How UNC-3 protein affects synapse development is presently not known.

Our screen appears to have identified putative novel genes involved in active zone development. Preliminary mapping data of some of the recovered mutants place them within regions that do not contain genes implicated previously in active zone formation (Table 1). Thus, SYD-2::GFP can be used as a tool to identify novel genes required specifically for synapse formation, including active zone assembly. We expect the molecular identification of these genes will help to elucidate the mechanisms that regulate the establishment of presynaptic active zones.

\section{Discussion}

We describe here a fluorescent active zone marker that can be used to visualize active zones in living C. elegans. In contrast to the localization of mammalian liprin- $\alpha$ family proteins, which appear to associate with both presynaptic and postsynaptic membranes in neuron cultures, SYD-2 is restricted to presynaptic active zones at both neuromuscular junctions and neuron-neuron synapses in $C$. elegans, serving as a protein marker for active zones. By creating a functional SYD-2::GFP fusion protein and expressing this marker in GABAergic motoneurons, we are able to visualize discrete puncta in living animals. Our data suggest that most of these puncta represent presynaptic active zone regions. We first demonstrated that at C. elegans synapses, SYD-2 is strictly presynaptic and restricted to active zone regions. We showed that endogenous SYD-2 colocalizes with other active zone components, UNC-10 and UNC-13. Furthermore, immunoelectron microscopy provided definitive evidence that SYD-2 tightly associates with the electron-dense presynaptic specializations at active zone regions. Next, we showed that SYD-2::GFP colocalizes with UNC-10 and always associates with SNB-1::CFP/DsRed vesicle puncta in living animals. Finally, SYD-2::GFP expression patterns in known synapse-defective mutants reflect their previously described defects in synapse distribution.

Vital markers by GFP tagging of confirmed or predicted active zone components, such as Bassoon, cacophony, and UNC-13, have been reported previously (Lackner et al., 1999; Nurrish et al., 1999; Friedman et al., 2000; Bresler et al., 2004; Kawasaki et al., 2004). GFP::Bassoon was used successfully for visualizing active zone packet transport and active zone development in transfected rat neuron cultures. GFP-tagged cacophony, a calcium channel $\alpha 1$ subunit and a predicted active zone component of Drosophila neuromuscular junctions, allowed for imaging in dissected animals. Although UNC-13 is an active zone component, the expression of the reported UNC-13::GFP marker does not appear punctate in neurons and therefore may not be restricted to presynaptic regions. SYD-2::GFP is the first fluorescent marker that can be used for both visualization and characterization of active zones in living animals.

Our studies to confirm the active zone localization of SYD-2 
yielded additional insights into synapse formation. Whereas most SYD-2 proteins colocalized with UNC-10 or UNC-13, some SYD-2-positive puncta did not appear to costain with UNC-10 or UNC-13. Although this may simply reflect a technical limit of double immunofluorescent staining, such as antibodies competing for a limited number of preserved and exposed epitopes in active zones, this may not be the sole explanation. We compared the results from either sequential or concomitant antibody staining and found that the ratio of costaining versus non-costaining puncta for SYD-2/UNC-10 remained similar. Some of these nonoverlapping puncta may actually represent a different abundance of active zone proteins at individual active zones. Another possibility is that not all SYD-2, UNC-10, or UNC-13 proteins are restricted to the synapse, even when they appear punctate. Multiple isoforms have been identified for UNC-10 and UNC-13, which may behave differently with respect to their localization at active zones. For example, it has been reported that the dynamic recruitment of the smaller isoform of UNC-13 protein to synapses during exocytosis occurs in C. elegans (Lackner et al., 1999). If so, individual active zones may possess different releasing properties based on the amounts of active zone components they contain.

We used the SYD-2::GFP marker to gain insight into the dynamics of synaptogenesis during C. elegans development. Previous studies documenting the connectivity and structure of the nematode nervous system have been done on adult animals and suggest that synapse number is consistent and relatively invariant between animals (White et al., 1986). Our studies support this conclusion, because the synapse number determined by either juIs1 or hpIs3 was consistent between animals. The new insight our studies suggest is that the total number of synapses, at least in GABAergic neuromuscular junctions, is established early in development and only increases slightly as the animals develop. Spacing of these synapses increases as the animals grow, resulting in a lower density of synapses along axons. In contrast to the relatively constant number of synapses made by GABAergic neurons, the size of vesicle clusters and the number of SYD-2::GFP puncta increase during development. The increasing ratio of SYD-2::GFP puncta to GABAergic synapses may reflect subsequent incorporation of active zone material at presynaptic termini. This would be consistent with an increase in the size or number of active zones, or an accumulation of specific protein components of active zones. These possibilities can only be verified directly by comparing active zone structures revealed by ultrastructural reconstruction of the $C$. elegans nervous system at earlier developmental stages.

The increasing ratio of SYD-2::GFP versus SNB-1::GFP is unlikely to be an artifact caused by variability or overexpression of specific Punc-25-SYD-2::GFP transgenic lines, because we examined several independent integrants, and all showed a similar number of puncta as $h p I s 3$ (data not shown). More importantly, multiple mutations have been found to disrupt the pattern in a distinctive manner, strongly suggesting that SYD-2::GFP localization in $h p I s 3$ animals is tightly regulated with specific mechanisms. Furthermore, the relatively constant density of SYD-2::GFP puncta during development is consistent with our observation that the density of endogenous SYD-2 and UNC-10 puncta remains constant along the dorsal cord throughout development. Most synapses along the dorsal cord are GABAergic and cholinergic neuromuscular junctions, suggesting a continuous increase in the total number of endogenous SYD-2 and UNC-10 puncta in those motoneurons during development. Thus, C. elegans GABAergic synapses may accommodate increasing body size mainly by increasing the size and releasing capac- ity of existing synapses. This hypothesis may be tested once electrophysiological recording on single synapses in C. elegans becomes possible. A previous study using a postsynaptic marker, GLR-1::GFP, suggested that glutamatergic synapses of $C$. elegans interneurons are generated and maintained at a constant density during development (Rongo and Kaplan, 1999). This is different from what we observe in GABAergic neurons. Specific neuronal subtypes may therefore use different mechanisms to accommodate the increasing innervated region.

Several mutants were recovered from our small-scale genetic screen, indicating the potential power of this marker to identify new genes involved in synapse formation. The fact that we were able to isolate new alleles of genes that were implicated previously in regulating synapse morphology $(r p m-1$, syd-1) and the recruitment of an active zone component ( goa-1) particularly strengthens the validation of this marker for analyzing active zone formation. The phenotype of SYD-2::GFP in goa-1 mutants may implicate a role of the Go $\alpha$ subunit in regulating the recruitment of multiple active zone components in addition to UNC-13. The identification of $u n c-3$ may also suggest a previously unidentified function of the UNC-3 transcription factor in synapse formation.

Preliminary studies have revealed that several of the identified mutants represent loci previously not implicated in synaptogenesis by other genetic or biochemical studies, including previous SNB-1::GFP screens. Although some of our mutants show abnormal behavior or response to pharmacological reagents such as aldicarb (data not shown), we have not found a distinct correlation between the severity or type of SYD-2::GFP mutant phenotype with any behavior or drug response defect. Therefore, our screen is unique in its ability to reveal mutants that may affect different aspects of synapse formation than the traditional behavior and drug-response screens. It was mentioned previously that vesicle markers such as SNB-1::GFP have been very useful in screens to identify new factors involved in synaptogenesis. However, SNB-1 protein expression and localization may preferentially reveal defects in machinery required for vesicle localization. SYD-2::GFP provides a marker allowing for the identification of genes that specifically affect active zone development. Even in the small-scale screen we performed, we have uncovered at least two mutations that disrupt the SYD-2::GFP pattern without severely affecting the SNB-1::GFP vesicle marker. This further demonstrates the potential power of the SYD-2::GFP marker to reveal mechanisms that specifically affect active zone formation during synaptogenesis that would not have been detected by SNB-1::GFP.

Another obvious use for this marker is to study syd-2/liprin function in detail. Although syd-2/liprin is clearly important for active zone and synapse formation in different species, little is known about the mechanism by which it regulates active zone morphology, and nothing is known about the dynamics of the protein. Although some of the identified mutants from our screen may affect active zone formation, some of them may also reflect defects in some aspect of SYD-2 protein function and/or localization. Identification of these factors will provide useful information regarding the molecular pathways by which syd-2 regulates active zone and synapse formation.

Recent studies in mouse CNS synapses suggest that some active zone proteins, such as Bassoon, Picollo, and RIM, are assembled in $80-n m$ dense-core vesicles and transported to presynaptic terminals to form active zones by quantal deposition of two to three of these active zone packets (Zhai et al., 2001). Mechanisms that regulate the biogenesis, transport, and fusion of these vesicles remain unclear. The mechanisms that drive the transport of other active zone proteins and the assembly of presynaptic active zone 
are also unknown. The SYD-2::GFP marker may provide a tool to investigate whether such a system of presynaptic delivery also exists in C. elegans. Our ongoing studies with the identified mutants may provide molecular insight into how C. elegans delivers presynaptic proteins to their targets.

\section{References}

Brenner S (1974) The genetics of Caenorhabditis elegans. Genetics 77:71-94. Bresler T, Shapira M, Boeckers T, Dresbach T, Futter M, Garner CC, Rosenblum K, Gundelfinger ED, Ziv NE (2004) Postsynaptic density assembly is fundamentally different from presynaptic active zone assembly. J Neurosci 24:1507-1520.

Broadie KS, Richmond JE (2002) Establishing and sculpting the synapse in Drosophila and C. elegans. Curr Opin Neurobiol 12:491-498.

Dresbach T, Qualmann B, Kessels MM, Garner CC, Gundelfinger ED (2001) The presynaptic cytomatrix of brain synapses. Cell Mol Life Sci 58:94-116.

Eastman C, Horvitz HR, Jin Y (1999) Coordinated transcriptional regulation of the unc-25 glutamic acid decarboxylase and the unc-47 GABA vesicular transporter by the Caenorhabditis elegans UNC-30 homeodomain protein. J Neurosci 19:6225-6234.

Friedman HV, Bresler T, Garner CC, Ziv NE (2000) Assembly of new individual excitatory synapses: time course and temporal order of synaptic molecule recruitment. Neuron 27:57-69.

Garner CC, Kindler S, Gundelfinger ED (2000) Molecular determinants of presynaptic active zones. Curr Opin Neurobiol 10:321-327.

Govind CK, Hirji R (2002) Free-floating active zone dense bar in a crab motor nerve terminal. Synapse 43:145-149.

Gustafsson JS, Birinyi A, Crum J, Ellisman M, Brodin L, Shupliakov O (2002) Ultrastructural organization of lamprey reticulospinal synapses in three dimensions. J Comp Neurol 450:167-182.

Hall DH, Hedgecock EM (1991) Kinesin-related gene unc-104 is required for axonal transport of synaptic vesicles in C. elegans. Cell 65:837-847.

Hallam SJ, Goncharov A, McEwen J, Baran R, Jin Y (2002) SYD-1, a presynaptic protein with PDZ, C2 and rhoGAP-like domains, specifies axon identity in C. elegans. Nat Neurosci 5:1137-1146.

Harlow ML, Ress D, Stoschek A, Marshall RM, McMahan UJ (2001) The architecture of active zone material at the frog's neuromuscular junction. Nature 409:479-484.

Jin Y (2002) Synaptogenesis: insights from worm and fly. Curr Opin Neurobiol 12:71-79.

Jin Y, Hoskins R, Horvitz HR (1994) Control of type-D GABAergic neuron differentiation by C. elegans UNC-30 homeodomain protein. Nature 372:780-783.

Jin Y, Jorgensen E, Hartwieg E, Horvitz HR (1999) The Caenorhabditis elegans gene unc- 25 encodes glutamic acid decarboxylase and is required for synaptic transmission but not synaptic development. J Neurosci 19:539-548.

Kaufmann N, DeProto J, Ranjan R, Wan H, Van Vactor D (2002) Drosophila liprin-alpha and the receptor phosphatase Dlar control synapse morphogenesis. Neuron 34:27-38.

Kawasaki F, Zou B, Xu X, Ordway RW (2004) Active zone localization of presynaptic calcium channels encoded by the cacophony locus of Drosophila. J Neurosci 24:282-285.

Kim S, Ko J, Shin H, Lee JR, Lim C, Han JH, Altrock WD, Garner CC, Gundelfinger ED, Premont RT, Kaang BK, Kim E (2003) The GIT family of proteins forms multimers and associates with the presynaptic cytomatrix protein Piccolo. J Biol Chem 278:6291-6300.

Ko J, Na M, Kim S, Lee JR, Kim E (2003) Interaction of the ERC family of RIM-binding proteins with the liprin-alpha family of multidomain proteins. J Biol Chem 278:42377-42385.

Koushika SP, Richmond JE, Hadwiger G, Weimer RM, Jorgensen EM, Nonet ML (2001) A post-docking role for active zone protein Rim. Nat Neurosci 4:997-1005.

Lackner MR, Nurrish SJ, Kaplan JM (1999) Facilitation of synaptic transmission by EGL-30 Gqalpha and EGL-8 PLCbeta: DAG binding to
UNC-13 is required to stimulate acetylcholine release. Neuron 24:335-346.

Liao EH, Hung W, Abrams B, Zhen M (2004) An SCF-like ubiquitin ligase complex that controls presynaptic differentiation. Nature 430:345-350.

McIntire SL, Jorgensen E, Horvitz HR (1993) Genes required for GABA function in Caenorhabditis elegans. Nature 364:334-337.

McIntire SL, Reimer RJ, Schuske K, Edwards RH, Jorgensen EM (1997) Identification and characterization of the vesicular GABA transporter. Nature 389:870-876.

Nonet ML (1999) Visualization of synaptic specializations in live C. elegans with synaptic vesicle protein-GFP fusions. J Neurosci Methods 89:33-40.

Nurrish S, Segalat L, Kaplan JM (1999) Serotonin inhibition of synaptic transmission: Galpha( 0$)$ decreases the abundance of UNC-13 at release sites. Neuron 24:231-242.

Otsuka AJ, Jeyaprakash A, Garcia-Anoveros J, Tang LZ, Fisk G, Hartshorne T, Franco R, Born T (1991) The C. elegans unc-104 gene encodes a putative kinesin heavy chain-like protein. Neuron 6:113-122.

Phillips GR, Huang JK, Wang Y, Tanaka H, Shapiro L, Zhang W, Shan WS, Arndt K, Frank M, Gordon RE, Gawinowicz MA, Zhao Y, Colman DR (2001) The presynaptic particle web: ultrastructure, composition, dissolution, and reconstitution. Neuron 32:63-77.

Prasad BC, Ye B, Zackhary R, Schrader K, Seydoux G, Reed RR (1998) unc-3, a gene required for axonal guidance in Caenorhabditis elegans, encodes a member of the O/E family of transcription factors. Development 125:1561-1568.

Richmond JE, Davis WS, Jorgensen EM (1999) UNC-13 is required for synaptic vesicle fusion in C. elegans. Nat Neurosci 2:959-964.

Rongo C, Kaplan JM (1999) CaMKII regulates the density of central glutamatergic synapses in vivo. Nature 402:195-199.

Rostaing P, Weimer RM, Jorgensen EM, Triller A, Bessereau JL (2004) Preservation of immunoreactivity and fine structure of adult C. elegans tissues using high-pressure freezing. J Histochem Cytochem 52:1-12.

Schaefer AM, Nonet ML (2001) Cellular and molecular insights into presynaptic assembly. Curr Opin Neurobiol 11:127-134.

Schaefer AM, Hadwiger GD, Nonet ML (2000) rpm-1, a conserved neuronal gene that regulates targeting and synaptogenesis in C. elegans. Neuron 26:345-356.

Schoch S, Castillo PE, Jo T, Mukherjee K, Geppert M, Wang Y, Schmitz F, Malenka RC, Sudhof TC (2002) RIMlalpha forms a protein scaffold for regulating neurotransmitter release at the active zone. Nature 415:321-326.

Serra-Pages C, Medley QG, Tang M, Hart A, Streuli M (1998) Liprins, a family of LAR transmembrane protein-tyrosine phosphatase-interacting proteins. J Biol Chem 273:15611-15620.

Shin H, Wyszynski M, Huh KH, Valtschanoff JG, Lee JR, Ko J, Streuli M, Weinberg RJ, Sheng M, Kim E (2003) Association of the kinesin motor KIF1A with the multimodular protein liprin-alpha. J Biol Chem 278:11393-11401.

White JG, Southgate E, Thomson JN, Brenner S (1986) The structure of the nervous system of the nematode Caenorhabditis elegans. Philos Trans R Soc Lond B Biol Sci 314:1-340.

Wyszynski M, Kim E, Dunah AW, Passafaro M, Valtschanoff JG, Serra-Pages C, Streuli M, Weinberg RJ, Sheng M (2002) Interaction between GRIP and liprin-alpha/SYD2 is required for AMPA receptor targeting. Neuron 34:39-52.

Yamagata M, Sanes JR, Weiner JA (2003) Synaptic adhesion molecules. Curr Opin Cell Biol 15:621-632.

Zhai RG, Vardinon-Friedman H, Cases-Langhoff C, Becker B, Gundelfinger ED, Ziv NE, Garner CC (2001) Assembling the presynaptic active zone: a characterization of an active one precursor vesicle. Neuron 29:131-143.

Zhen M, Jin Y (1999) The liprin protein SYD-2 regulates the differentiation of presynaptic termini in C. elegans. Nature 401:371-375.

Zhen M, Jin Y (2004) Presynaptic terminal differentiation: transport and assembly. Curr Opin Neurobiol 14:280-287.

Zhen M, Huang X, Bamber B, Jin Y (2000) Regulation of presynaptic terminal organization by C. elegans RPM-1, a putative guanine nucleotide exchanger with a RING-H2 finger domain. Neuron 26:331-343. 